\title{
Association of Albuminuria and Estimated Glomerular Filtration Rate with Functional Performance Measures in Older Adults with Chronic Kidney Disease
}

\author{
Dawn F. Wolfgram ${ }^{a, c}$ Katelyn Garciae Greg Evans ${ }^{e}$ Sara Zamanian $^{f}$ \\ Rocky Tang ${ }^{g}$ Thomas Wiegmann $^{\mathrm{h}}$ Rajeev Sharma ${ }^{\mathrm{i}}$ Ruth Campbell ${ }^{j}$ \\ Jeff Whittle ${ }^{b, d}$ for the SPRINT Study Research Group \\ a Medicine Division, Section of Nephrology, and b Primary Care, Milwaukee VA Medical Center, \\ 'Department of Medicine, Division of Nephrology, and d Department of Medicine, Division of General \\ Internal Medicine, Medical College of Wisconsin, Milwaukee, WI, ${ }^{e}$ Department of Biostatistical Sciences, \\ Wake Forest University Health Sciences, Winston-Salem, NC, ${ }^{\mathrm{f}}$ Department of Medicine, Case Western \\ Reserve University, Cleveland, $\mathrm{OH},{ }^{9}$ Department of Surgery, Columbia University, New York, NY, \\ ${ }^{\mathrm{h}}$ Research Service, Kansas City VA Medical Center, Kansas City, MO, 'Department of Medicine, Division of \\ Endocrinology, State University of New York Downstate Medical Center, Brooklyn, NY, and ${ }^{j}$ Department of \\ Medicine, Medical University of South Carolina, Charleston, SC, USA
}

\section{Keywords}

Functional impairment · Albuminuria · Chronic kidney disease

\begin{abstract}
Background: Chronic kidney disease (CKD) is increasingly common and disproportionately affects older adults. The contribution of kidney disease to the functional impairment noted in the elderly CKD population is unclear. Methods: This is a cross-sectional analysis of a hypertensive cohort of people aged $\geq 75$ years from the Systolic Blood Pressure Intervention Trial. We evaluated estimated glomerular filtration rate (eGFR) and urine albumin-to-creatinine ratio (UACR) as predictors of 3 measures of functional status: EuroQol-5 Dimensional (EQ-5D) score, Falls Efficacy Scale (FES) score, and gait speed. Linear regression models were used to evaluate the associations between our independent variables
\end{abstract}

and outcome measures. Results: Our analysis included 2,620 participants, mean age of 79.9 (4.0) years. Unadjusted models showed that lower eGFR level and higher UACR level were associated with lower EQ-5D ( $p<0.001$ for both) and slower gait speed ( $p<0.001$ for both) and worse scores on FES ( $p=0.032$ and $p=0.039$ ). In the fully adjusted models, higher levels of UACR remained significantly associated with lower EQ-5D scores and slower gait speed ( $p=0.011$ and $p=$ 0.002 , respectively). In contrast, level of eGFR was not associated with any functional outcome measures when accounting for covariates. Conclusions: In individuals aged $\geq 75$ years, albuminuria and eGFR were associated with impairments in physical performance and self-reported functional status; however, only the association with albuminuria remained after adjusting for relevant demographics and comorbidities. Evaluation of albuminuria may provide an additional tool for identifying older individuals at risk for functional impairment.

\section{KARGER}

(c) 2017 S. Karger AG, Basel

E-Mail karger@karger.com

www.karger.com/ajn
Dawn F. Wolfgram, MD

Milwaukee VA Medical Center

5000 West National Ave CC-111K

Milwaukee, WI 53295-1000 (USA)

E-Mail dwolfgram@mcw.edu 


\section{Introduction}

Chronic kidney disease (CKD) is increasingly common in the US population, especially in older adults. It is estimated that $14 \%$ of the US population has CKD; that prevalence rises to $35 \%$ in adults over 60 years of age [1]. CKD is associated with adverse outcomes including death and cardiovascular disease (CVD), with increased risk present for individuals with early CKD as well as end-stage renal disease (ESRD) [2]. Despite its importance, research on the contribution of CKD to health-related quality of life outcomes, such as functional status, has been far less extensive. While the association of ESRD with functional status is established [3-5], it is not clear that less advanced CKD affects function, independent of its association with other chronic conditions. It is important to clarify if CKD is associated with impaired functional status, which is associated with increased healthcare utilization and mortality. This is particularly important for older patients, who make up the majority of Americans with CKD. Aging itself is a risk factor for functional impairment, thus older adults with CKD may be at greater risk for functional impairment. Additionally, older adults with CKD are at higher risk for subsequent decline in activities of daily living and community mobility compared to those without CKD $[6,7]$.

Studies evaluating functional impairment in patients with CKD have been limited by small cohorts, often special populations (i.e., women only) and few older participants $[8,9]$. In addition, few have examined gradations of estimated glomerular filtration rate (eGFR) levels, focusing more on the cutoff of eGFR $<60 \mathrm{~mL} /$ $\mathrm{min} / 1.73 \mathrm{~m}^{2}$. Other analyses, including the recent Framingham Offspring Study, have focused on the development of functional impairment in subsequent years in persons who have CKD at baseline $[10,11]$. Those studies do not give us a clear understanding of CKD status and functional impairment since the level of renal function at the time that functional impairment was noted was not described. Furthermore, prior research on this subject has not evaluated the role of albuminuria, a determinant of CKD severity, in functional status. We therefore examined the cohort of older patients with hypertension enrolled in the Systolic Blood Pressure Intervention Trial (SPRINT) for evidence of an association between markers of CKD severity and various domains of functional status including physical performance, activity limitations, and concern for falls.

Albuminuria and Functional Status

\section{Methods}

Design

SPRINT was a multicenter randomized clinical trial sponsored by the National Heart, Lung, and Blood Institute (NHLBI), the National Institute for Diabetes and Digestive and Kidney Diseases, National Institute on Aging (NIA), and the National Institute of Neurological Disorders and Stroke (NINDS). A thorough description of the rationale and design of SPRINT has been published [12]. In brief, the goal of SPRINT was to determine if treatment targeting a systolic blood pressure (SBP) $<120 \mathrm{~mm} \mathrm{Hg}$ compared to targeting SBP $<140 \mathrm{~mm} \mathrm{Hg}$ would reduce CVD events. SPRINT was approved by Institutional Review Boards at participating sites and all participants provided written informed consent.

\section{Participants}

The senior SPRINT subgroup, which consisted of individuals aged $\geq 75$ years of age at enrollment, was included in this analysis. The senior subgroup was one of the 3 subgroups targeted during enrollment. The complete SPRINT cohort was made up of participants aged $\geq 50$ years and at risk for cardiovascular event based on either clinical CVD or subclinical CVD or high risk for CVD, including eGFR $25-59 \mathrm{~mL} / \mathrm{min} / 1.73 \mathrm{~m}^{2}$ or Framingham risk score for 10 year CVD risk $\geq 15 \%$. SPRINT exclusion criteria included a known history of stroke, diabetes mellitus, $>600 \mathrm{mg}$ /day of albuminuria, or $>1$ $\mathrm{g} /$ day of proteinuria based on $24 \mathrm{~h}$ collection or if not available a spot urine ratio within the last 6 month, or eGFR $<20 \mathrm{~mL} / \mathrm{min} / 1.73 \mathrm{~m}^{2}$.

\section{Data Collection}

At the randomization visit, participants completed a self-administered survey, including demographic data. Other items included socioeconomic status, tobacco and alcohol use, self-reported medical conditions, and the EuroQol-5 Dimensional (EQ-5D) measure of health-related quality of life. At this same visit, SPRINT staff measured height, weight, and blood pressure, performed a brief physical examination including a timed $4 \mathrm{~m}$ walk to measure gait speed for those aged $\geq 75$ years, collected urine and blood for laboratory analysis, and recorded an electrocardiogram. A random subset of participants plus all seniors randomized after January 8 , 2013, also completed the Falls Efficacy Scale (FES).

\section{Outcome Measures}

We used 3 measures to gain a comprehensive view of functional status: the EQ-5D, FES, and gait speed. We used the EQ-5D to measure health-related quality of life concentrating on functional status. It includes 5 questions on mobility, ability to perform selfcare, ability to perform usual daily activities, pain/discomfort, and anxiety/depression. EQ-5D is commonly used to measure healthrelated quality of life; however, it focuses on function in 3 out of the 5 questions and pain level is also likely to influence functional status. We used standard methods to summarize the EQ-5D, with scores ranging from -0.11 (i.e., worse than death) to 1.0 (perfect health) [13]. The FES includes 7 items that are scored on a 4-point Likert scale and summarized by simple addition to yield a score ranging from 7 to 28 with higher scores indicating increased concern for falls [14]. Increased scores are associated with falls, decreased functional activity, and limitations in social activity [15, 16]. We used gait speed as a measure of physical performance. Population studies show normal gait speeds range from 0.78 to $0.96 \mathrm{~m} / \mathrm{s}$ among individuals aged $>70$ years [17]. 
Measures of CKD

The SPRINT Central Lab measured creatinine in serum or urine on a Roche Modular P Chemistry Analyzer (Roche Diagnostics Corporation) using a creatinase enzymatic method, which has been standardized against the isotope dilution mass spectrometry procedure. Urine albumin was measured by nephelometry using the Siemens ProSpec nephelometer and a rabbit-derived anti-human albumin antibody. We calculated the urine albumin/creatinine ratio (UACR) using a spot urine sample obtained at the baseline visit and measured in milligrams of albumin/gram of creatinine $(\mathrm{mg} / \mathrm{g})$. We calculated eGFR in $\mathrm{mL} / \mathrm{min} / 1.73 \mathrm{~m}^{2}$ using the serum creatinine level obtained at the baseline visit. We used the 4 -variable MDRD equation for our primary analysis and repeated the analysis after recalculating eGFR using the Chronic Kidney Disease Epidemiology Collaboration equation; the results were similar for the fully adjusted models [18].

\section{Statistical Analysis}

We present baseline characteristics as means (SD) or frequencies, unless otherwise noted. We compared baseline characteristics between persons with eGFR $\leq 60$ vs. $>60 \mathrm{~mL} / \mathrm{min} / 1.73 \mathrm{~m}^{2}$ using 2 sample $t$ tests for continuous variables and chi-square tests for categorical variables. We then used linear regression models to evaluate the cross-sectional associations between our independent variables and outcome measures. Indicator variables were used to model categories of eGFR $\left(<44,44-59\right.$, and $\left.>60 \mathrm{~mL} / \mathrm{min} / 1.73 \mathrm{~m}^{2}\right)$ and UACR $(\leq 30$, 31-299, and $>300 \mathrm{mg} / \mathrm{g}$ ). The first model adjusted for eGFR or UACR categories without further adjustment. Additional models adjusted for demographic covariates (age, gender, race, body mass index, smoking, and drinking status), and comorbidities (coronary artery disease, congestive heart failure, peripheral vascular disease, arthritis, low back pain, depression, SBP and diastolic blood pressures, and orthostatic hypotension). The final model also included UACR for the eGFR-based model and eGFR for the UACR-based model. Adjusted means for the levels of eGFR and UACR were calculated as part of the multivariable analyses. Diagnostic tests and graphics were completed for all model assumptions. All statistical analyses were performed using SAS 9.4 (SAS Institute, Cary, NC, USA).

\section{Results}

SPRINT investigators enrolled 9,361 participants at 102 clinics over a 2.3 year period. After applying age exclusion, our study population included 2,620 participants, most of whom were male (62\%). Their mean age was 79.9 (4.0) years. See Table 1 for baseline characteristics of the cohort. As intended, participants included large numbers of individuals who had CKD $(n=1,161,44 \%)$. In our cohort, the median (25th-75th percentile) eGFR was 62.54 $\mathrm{mL} / \mathrm{min} / 1.73 \mathrm{~m}^{2}(50.70-75.22)$ and the median (25th75th percentile) UACR was $13.16 \mathrm{mg} / \mathrm{g}(7.24-32.35)$.

\section{EuroQol-5 Dimensional}

The mean (SD) EQ-5D score was 0.85 (0.13). In the unadjusted analysis (Model 1), mean EQ-5D scores were similar amongst participants with an eGFR $>44$ to $<60$ and $\geq 60$ and were slightly higher compared to the scores of participants with eGFR $\leq 44(p<0.001$; Table 2$)$. EQ$5 \mathrm{D}$ scores declined as level of albuminuria increased $(p<$ 0.001; Table 3). After adjustment for baseline demographics as potential confounders (Model 2), both lower eGFR and higher UACR remained associated with lower EQ-5D scores $(p=0.007$ and $p<0.001$; Tables 2,3$)$. However, after fully adjusting for demographics and comorbidities (Model 3), eGFR was no longer associated with EQ-5D score $(p=0.443)$ while UACR remained significantly associated ( $p=0.040$; Tables 2,3 , respectively). Graphical results of Model 3 are available online as online supplemental Figure 1 (for all online suppl. material, see www.karger.com/doi/10.1159/000455388).

\section{Gait Speed}

The mean (SD) gait speed was $0.87(0.22) \mathrm{m} / \mathrm{s}$. In the unadjusted analysis (Model 1), mean gait speed increased among participants as eGFR increased $(p<0.001)$ and decreased as UACR increased ( $p<0.001$; Tables 2,3 , respectively). After adjustment for baseline demographics (Model 2) and comorbidities (Model 3), level of eGFR was no longer associated with gait speed $(p=0.063$ and $p=0.520)$. UACR remained significantly associated with gait speed after adjusting for the additional demographics (Model 2) and comorbidities (Model 3; $p<0.001$ and $p=$ 0.003 ; Table 3). Graphical results of Model 3 are available online as online supplemental Figure 2.

\section{Falls Efficacy Scale}

The mean (SD) FES score was 9.12 (3.34). In the unadjusted analysis (Model 1), higher (worse) FES scores were associated with lower level eGFR $(p=0.032)$ and higher UACR ( $p=0.039$; Tables 2,3 , respectively). The associations with eGFR and FES scores did not persist in the adjusted models (Model 2, $p=0.173$; Model 3, $p=0.181$ ). The association between UACR and FES scores remained after adjusting for baseline demographics ( $p=0.020$; Model 2) but not when including comorbidities ( $p=0.122$; Model 3). Graphical results of Model 3 are available online as online supplemental Figure 3.

\section{Discussion}

In a cohort of older adults with hypertension, we found that increased level of albuminuria was associated with slower gait speed, a measure of physical function, and worse EQ-5D score, a measure of health-related quality 
Table 1. Baseline characteristics of SPRINT cohort by CKD status

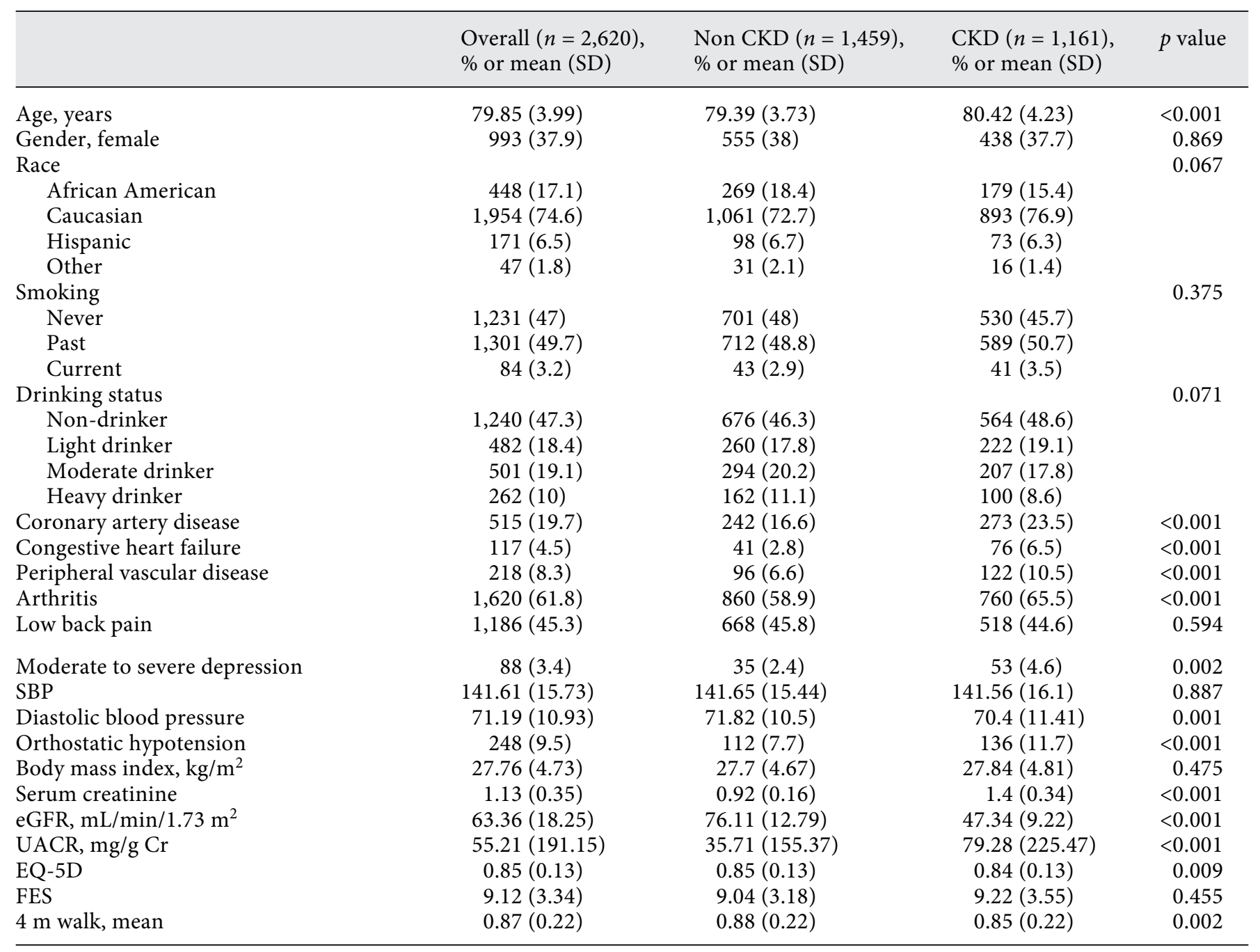

* CKD based on eGFR $<60 \mathrm{~mL} / \mathrm{min} / 1.73 \mathrm{~m}^{2}$, eGFR, estimated glomerular filtration rate.

of life with a focus on function. This association remained even after adjusting for relevant covariates. In contrast, the level of eGFR was associated with our functional outcome measures when unadjusted; however, after adjusting for relevant covariates, the associations were no longer significant. Our study suggests that kidney disease as noted by increased level of albuminuria is associated with functional impairment in older adults.

Our study adds to the evidence that in persons with $\mathrm{CKD}$, there are impairments in function. However, in contrast to previous research, we did not find that eGFR level (above $20 \mathrm{~mL} / \mathrm{min} / 1.73 \mathrm{~m}^{2}$ ) alone was associated with functional impairment after adjusting for demographics and relevant covariates. We note that previous research demonstrating an association of eGFR and functional status was in select patient populations, such as patients with coronary artery disease, a disease which may modify susceptibility to functional impairment $[8,9]$. Furthermore, we evaluated the association between current eGFR and functional impairment in contrast to other studies that evaluated the subsequent development of functional impairment associated with a baseline eGFR $[10,11]$. Evaluating the subsequent development of functional limitations does not provide the severity of CKD at the time of impaired function as CKD may have progressed during follow-up. Additionally, previous studies did not include albuminuria as a risk factor for functional impairment. Since decline in eGFR is higher in those 
Table 2. EQ-5D, mean gait speed, and FES (adjusted means [SE]) at baseline by eGFR categories

\begin{tabular}{|c|c|c|c|c|}
\hline & \multicolumn{3}{|c|}{$\mathrm{eGFR}, \mathrm{mL} / \mathrm{min} / 1.73 \mathrm{~m}^{2}$} & \multirow[t]{2}{*}{$p$ value } \\
\hline & $\leq 44$ & $>44-<60$ & $\geq 60$ & \\
\hline $\mathrm{EQ}-5 \mathrm{D}, n$ & 372 & 781 & 1,449 & \\
\hline Model 1 & $0.82(0.01)$ & $0.85(0.01)$ & $0.85(0.00)$ & $<0.001$ \\
\hline Model 2 & $0.82(0.01)$ & $0.84(0.01)$ & $0.84(0.01)$ & 0.007 \\
\hline Model 3 & $0.73(0.01)$ & $0.75(0.01)$ & $0.73(0.01)$ & 0.443 \\
\hline Mean gait speed, $n$ & 355 & 755 & 1,392 & \\
\hline Model 1 & $0.83(0.01)$ & $0.86(0.01)$ & $0.88(0.01)$ & $<0.001$ \\
\hline Model 2 & $0.77(0.02)$ & $0.79(0.01)$ & $0.80(0.01)$ & 0.063 \\
\hline Model 3 & $0.74(0.02)$ & $0.75(0.02)$ & $0.76(0.02)$ & 0.520 \\
\hline FES, $n$ & 109 & 253 & 495 & \\
\hline Model 1 & $9.89(0.34)$ & $8.93(0.21)$ & $9.04(0.15)$ & 0.032 \\
\hline Model 2 & $9.95(0.48)$ & $9.23(0.39)$ & $9.42(0.36)$ & 0.173 \\
\hline Model 3 & $11.08(0.59)$ & $10.54(0.56)$ & $10.93(0.51)$ & 0.181 \\
\hline
\end{tabular}

Model 1: unadjusted.

Model 2: model 1 with age, gender, race, body mass index, smoking status, and drinking status.

Model 3: model 2 plus coronary artery disease, congestive heart failure, peripheral vascular disease, arthritis, low back pain, depression, SBP, diastolic blood pressure, DBP, orthostatic hypotension, and UACR.

Table 3. EQ-5D, mean gait speed, and FES (adjusted means [SE]) at baseline by UACR categories

\begin{tabular}{|c|c|c|c|c|}
\hline & \multicolumn{3}{|c|}{ UACR, mg/g Cr } & \multirow[t]{2}{*}{$p$ value } \\
\hline & $\leq 30$ & $>30-<300$ & $\geq 300$ & \\
\hline EQ-5D, $n$ & 1,846 & 566 & 91 & \\
\hline Model 1 & $0.85(0.00)$ & $0.84(0.01)$ & $0.81(0.01)$ & $<0.001$ \\
\hline Model 2 & $0.84(0.01)$ & $0.83(0.01)$ & $0.80(0.02)$ & $<0.001$ \\
\hline Model 3 & $0.74(0.01)$ & $0.74(0.01)$ & $0.71(0.02)$ & 0.040 \\
\hline Mean gait speed, $n$ & 1,782 & 540 & 85 & \\
\hline Model 1 & $0.88(0.01)$ & $0.83(0.01)$ & $0.84(0.02)$ & $<0.001$ \\
\hline Model 2 & $0.81(0.01)$ & $0.77(0.02)$ & $0.77(0.03)$ & $<0.001$ \\
\hline Model 3 & $0.77(0.02)$ & $0.74(0.02)$ & $0.74(0.03)$ & 0.003 \\
\hline FES, $n$ & 615 & 185 & 33 & \\
\hline Model 1 & $9.04(0.13)$ & $9.09(0.24)$ & $10.55(0.57)$ & 0.039 \\
\hline Model 2 & $9.19(0.37)$ & $8.94(0.41)$ & $10.61(0.63)$ & 0.020 \\
\hline Model 3 & $10.65(0.52)$ & $10.33(0.53)$ & $11.49(0.69)$ & 0.122 \\
\hline
\end{tabular}

Model 1: unadjusted.

Model 2: model 1 with age, gender, race, body mass index, smoking status, and drinking status.

Model 3: model 2 plus coronary artery disease, congestive heart failure, peripheral vascular disease, arthritis, low back pain, depression, SBP, diastolic blood pressure, DBP, orthostatic hypotension, and eGFR.

with increased albuminuria [19], it is possible that previous findings, similar to our results, actually reflected the importance of albuminuria in functional outcomes. Finally, the lack of association with eGFR may be due to the decreased accuracy of creatinine-based GFR estimates in the elderly [20]. In our cohort, the severity of CKD may not be accurately reflected with our creatinine-based eGFR in those aged $\geq 75$ years.

While albuminuria is known to relate to severity and prognosis of $\mathrm{CKD}$, our results indicate it may also be a marker for decreased functional status, at least in a hypertensive population without diabetes. In a recent report, a 
higher degree of albuminuria but not eGFR was associated with increased risk for serious falls among Medicare beneficiaries aged $\geq 65$ years, even after adjustment for demographic, comorbid, and fall risk factors [21]. This is consistent with our results as slower gait speed is associated with increased falls risk [22]. Furthermore, in two large trials focused on participants with diabetes or vascular disease, albuminuria was associated with worse performance on cognitive assessments at baseline and in follow-up $[23,24]$. These authors hypothesized that microvascular pathology could lead to both albuminuria and cerebral white matter disease. Similarly, our results could reflect that albuminuria is a marker of vascular endothelial pathology that is associated with the increased inflammatory state of CKD. Such microvascular pathology could also lead to skeletal muscle damage and impaired physical performance. Observational analysis has demonstrated that persons with albuminuria have a higher risk for sarcopenia, further supporting a link between physical performance and albuminuria [25].

If our results are confirmed in other studies, they suggest that screening older adults with kidney disease marked by macroalbuminuria for impaired physical function could improve outcomes. For example, if screening identified reduced or rapidly declining gait speed, physical therapy could be used to reduce falls and maintain mobility and independence [26, 27].

The strengths of our study include our relevant measures of both patient reported functional status and objectively assessed physical performance along with a large cohort of older adults. Our physical performance measure is clinically important as gait speed has already been shown to be a predictor of adverse outcomes in elderly individuals, including functional declines [28], hospitalizations $[29,30]$, falls [22], and mortality [30-32]. A decrease of 0.1 $\mathrm{m} / \mathrm{s}$ in gait speed is associated with a $6-17 \%$ decrease in survival depending on age stratum [32]. The $0.03 \mathrm{~m} / \mathrm{s}$ difference in gait speed that we observed between SPRINT participants with macroalbuminuria and those with normoalbuminuria is similar to changes in gait speed that have been reported to have a meaningful impact on patient reported mobility including the ability to walk a quarter mile, and ability to climb one flight of stairs [33, 34]. Using gait speed, we were able to identify subtle differences in functional status that may progress and lead to disability and the adverse outcomes described above. Our patient reported outcome, the EQ-5D questionnaire, is a widely used and validated measure of health-related quality of life with a focus on function $[35,36]$. In addition to the above outcomes, we also included a measure of the patients' perception of risk of falling while performing routine daily activities (FES). Overall, our cohort displayed only mild concern for falls with the average score of 9 [14]. From our FES results, we were able to conclude that there was no increased concern for falls at early to moderate stages of CKD. Thus, the decrease in gait speed associated with increased albuminuria is unlikely to be attributed to an increased concern for fall or patient selfimposed limitations but may reflect a more direct effect of kidney disease on physical capabilities. This adds evidence for the importance of screening older adults with albuminuria for physical impairment, since they may not be aware of impairment or think to report the impairment until it has progressed to a more severe stage.

Our study has the following limitations. First, we cannot determine whether the association we observed between albuminuria and reduced functional status is causal. Increased albuminuria due to primary renal disease could lead to impaired function or another disease process that could both cause albuminuria and impair function status. Second, the number of participants with UACR $\geq 300 \mathrm{mg} / \mathrm{g}$ and $\mathrm{eGFR} \leq 44 \mathrm{~mL} / \mathrm{min} / 1.73 \mathrm{~m}^{2}$ was small and participants with albuminuria $>600 \mathrm{mg} / \mathrm{g}$ were excluded, limiting our ability to determine if greater levels of albuminuria or lower eGFR would be associated with worse functional outcomes. Third, we did not extensively evaluate other clinical variables commonly seen in early kidney disease such as abnormalities in bone mineral parameters. Fourth, our cohort had participants in a clinical trial with existing CVD or risk factors for CVD, which affects its applicability to the general population. Finally, the differences we observed between different levels of albuminuria are small. Our fully adjusted results show that the EQ-5D score was 0.03 points lower among participants with macroalbuminuria than among those with normoalbuminuria. Although a meaningful difference in EQ-5D score varies based on disease state and age, the difference that we note is similar to changes seen with 10 -year increases in age according to published US population norms [37]. Similarly, the difference in gait speed we observed is associated with small yet meaningful changes in patient reported mobility $[33,34]$.

\section{Conclusion}

We evaluated a range of functional outcomes in older adults with and without markers of CKD. Our results indicate that among older hypertensive adults, albuminuria is associated with impairments in functional status. 
As SPRINT final closeout visits are completed and the data becomes available, the longitudinal relationship between albuminuria and functional status can be evaluated. Future research should confirm this association in a broader population and explore the relationship between functional impairment and other clinical variables noted in mild to moderate kidney disease. This may help identify both the appropriate time point for intervention and potential therapeutic interventions for the functional impairment noted in older adults with kidney disease.

\section{Acknowledgments}

The SPRINT is funded with Federal funds from the National Institutes of Health (NIH), including the NHLBI, the National Institute of Diabetes and Digestive and Kidney Diseases, the NIA, and the NINDS, under contract numbers HHSN268200900040C, HHSN268200900046C,HHSN268200900047C,HHSN268200900048C, HHSN268200900049C, and Inter-Agency Agreement Number A-HL-13-002-001. It was also supported in part with resources and use of facilities through the Department of Veterans Affairs. The SPRINT investigators acknowledge the contribution of study medications (azilsartan and azilsartan combined with chlorthalidone) from Takeda Pharmaceuticals International, Inc. All components of the SPRINT study protocol were designed and implemented by the investigators. The investigative team collected, analyzed, and interpreted the data. All aspects of manuscript writing and revision were carried out by the coauthors. The content is solely the responsibility of the authors and does not necessarily represent the official views of the NIH, the US Department of Veterans Affairs, or the United States Government. For a full list of contributors to SPRINT, please see the online supplementary acknowledgement list.

\section{SPRINT Acknowledgment}

We also acknowledge the support from the following CTSAs funded by NCATS: CWRU: UL1TR000439, OSU: UL1RR025755, U Penn: UL1RR024134 and UL1TR000003, Boston: UL1RR025771, Stanford: UL1TR000093, Tufts: UL1RR025752, UL1TR000073, and UL1TR001064, University of Illinois: UL1TR000050, University of Pittsburgh: UL1TR000005, UT Southwestern: 9U54TR000017-06, University of Utah: UL1TR000105-05, Vanderbilt University: UL1 TR000445, George Washington University: UL1TR000075, University of CA, Davis: UL1 TR000002, University of Florida: UL1 TR000064, University of Michigan: UL1TR000433, Tulane University: P30GM103337 COBRE Award NIGMS.

\section{Disclosure Statement}

The authors have no conflicts of interest to declare.

\section{References}

1 US Renal Data System: USRDS 2015 Annual Data Report: Atlas of Chronic Kidney Disease and End-Stage Renal Disease in the United States. Bethesda, National Institutes of Health, National Institute of Diabetes and Digestive and Kidney Diseases, 2015.

2 Go AS, Chertow GM, Fan D, McCulloch CE, Hsu CY: Chronic kidney disease and the risks of death, cardiovascular events, and hospitalization. N Engl J Med 2004;351:1296-1305.

3 Kurella Tamura M, Covinsky KE, Chertow GM, Yaffe K, Landefeld CS, McCulloch CE: Functional status of elderly adults before and after initiation of dialysis. N Engl J Med 2009; 361:1539-1547.

4 Cook WL, Jassal SV: Functional dependencies among the elderly on hemodialysis. Kidney Int 2008;73:1289-1295.

5 Gutman RA, Stead WW, Robinson RR: Physical activity and employment status of patients on maintenance dialysis. $\mathrm{N}$ Engl J Med 1981;304:309-313.

6 Bowling CB, Sawyer P, Campbell RC, Ahmed A, Allman RM: Impact of chronic kidney disease on activities of daily living in community-dwelling older adults. J Gerontol A Biol Sci Med Sci 2011;66:689-694.

7 Bowling CB, Muntner P, Sawyer P, Sanders PW, Kutner N, Kennedy R, et al: Community mobility among older adults with reduced kidney function: a study of life-space. Am J Kidney Dis 2014;63:429-436.

8 Kurella M, Ireland C, Hlatky MA, Shlipak MG, Yaffe K, Hulley SB, Chertow GM: Physi$\mathrm{cal}$ and sexual function in women with chronic kidney disease. Am J Kidney Dis 2004;43: 868-876.

9 Odden MC, Whooley MA, Shlipak MG: Association of chronic kidney disease and anemia with physical capacity: the heart and soul study. J Am Soc Nephrol 2004;15:29082915.

10 Fried LF, Lee JS, Shlipak M, Chertow GM, Green C, Ding J, et al: Chronic kidney disease and functional limitation in older people: health, aging and body composition study. J Am Geriatr Soc 2006;54:750-756.

11 Liu CK, Lyass A, Massaro JM, D’Agostino RB Sr, Fox CS, Murabito JM: Chronic kidney disease defined by cystatin $\mathrm{C}$ predicts mobility disability and changes in gait speed: the Framingham Offspring Study. J Gerontol A Biol Sci Med Sci 2014;69:301-307.

12 Ambrosius WT, Sink KM, Foy CG, Berlowitz DR, Cheung AK, Cushman WC, et al: The design and rationale of a multicenter clinical trial comparing two strategies for control of systolic blood pressure: the Systolic Blood
Pressure Intervention Trial (SPRINT). Clin Trials 2014;11:532-546.

13 Calculating the US Population-Based EQ-5D Index Score. Rockville, Agency for Healthcare Research and Quality, 2005. https://www. ahrq.gov/rice/ED5Dscore.htm.

14 Kumar A, Carpenter H, Morris R, Iliffe $S$, Kendrick D: Which factors are associated with fear of falling in community-dwelling older people? Age Ageing 2014;43:7684.

15 Friedman SM, Munoz B, West SK, Rubin GS, Fried LP: Falls and fear of falling: which comes first? A longitudinal prediction model suggests strategies for primary and secondary prevention. J Am Geriatr Soc 2002;50:13291335.

16 Scheffer AC, Schuurmans MJ, van Dijk N, van der Hooft T, de Rooij SE: Fear of falling: measurement strategy, prevalence, risk factors and consequences among older persons. Age Ageing 2008;37:19-24.

17 Bohannon RW: Population representative gait speed and its determinants. J Geriatr Phys Ther 2008;31:49-52.

18 Levey AS, Stevens LA, Schmid CH, Zhang YL, Castro AF 3rd, Feldman HI, et al: A new equation to estimate glomerular filtration rate. Ann Intern Med 2009;150:604-612. 
19 Ruggenenti P, Perna A, Mosconi L, Matalone M, Pisoni R, Gaspari F, et al: Proteinuria predicts end-stage renal failure in non-diabetic chronic nephropathies. The "Gruppo Italiano di Studi Epidemiologici in Nefrologia" (GISEN). Kidney Int Suppl 1997;63:S54-S57.

20 Kilbride HS, Stevens PE, Eaglestone G, Knight S, Carter JL, Delaney MP, et al: Accuracy of the MDRD (Modification of Diet in Renal Disease) study and CKD-EPI (CKD Epidemiology Collaboration) equations for estimation of GFR in the elderly. Am J Kidney Dis 2013;61:57-66.

21 Bowling CB, Bromfield SG, Colantonio LD, Gutierrez OM, Shimbo D, Reynolds K, et al: Association of reduced eGFR and albuminuria with serious fall injuries among older adults. Clin J Am Soc Nephrol 2016;11:12361243.

22 Montero-Odasso M, Schapira M, Soriano ER, Varela M, Kaplan R, Camera LA, et al: Gait velocity as a single predictor of adverse events in healthy seniors aged 75 years and older. J Gerontol A Biol Sci Med Sci 2005;60:13041309.

23 Murray AM, Barzilay JI, Lovato JF, Williamson JD, Miller ME, Marcovina S, et al: Biomarkers of renal function and cognitive impairment in patients with diabetes. Diabetes Care 2011;34:1827-1832.

24 Barzilay JI, Gao P, O’Donnell M, Mann JF, Anderson C, Fagard R, et al: Albuminuria and decline in cognitive function: the ONTAR-
GET/TRANSCEND studies. Arch Intern Med 2011;171:142-150.

25 Kim TN, Lee EJ, Hong JW, Kim JM, Won JC, Kim MK, et al: Relationship between sarcopenia and albuminuria: the 2011 Korea national health and nutrition examination survey. Medicine (Baltimore) 2016;95:e2500.

26 Judge JO: Balance training to maintain mobility and prevent disability. Am J Prev Med 2003;25(3 suppl 2):150-156.

27 Varma VR, Hausdorff JM, Studenski SA, Rosano C, Camicioli R, Alexander NB, et al: Aging, the central nervous system, and mobility in older adults: interventions. J Gerontol A Biol Sci Med Sci 2016;71:1451-1458.

28 Guralnik JM, Ferrucci L, Pieper CF, Leveille SG, Markides KS, Ostir GV, et al: Lower extremity function and subsequent disability: consistency across studies, predictive models, and value of gait speed alone compared with the short physical performance battery. J Gerontol A Biol Sci Med Sci 2000;55:M221M231.

29 Studenski S, Perera S, Wallace D, Chandler JM, Duncan PW, Rooney E, et al: Physical performance measures in the clinical setting. J Am Geriatr Soc 2003;51:314-322.

30 Cesari M, Kritchevsky SB, Penninx BW, Nicklas BJ, Simonsick EM, Newman AB, et al: Prognostic value of usual gait speed in wellfunctioning older people - results from the health, aging and body composition study. J Am Geriatr Soc 2005;53:1675-1680.
31 Koufaki P, Kouidi E: Current best evidence recommendations on measurement and interpretation of physical function in patients with chronic kidney disease. Sports Med 2010;40:1055-1074.

32 Studenski S, Perera S, Patel K, Rosano C, Faulkner K, Inzitari M, et al: Gait speed and survival in older adults. JAMA 2011;305:50-58.

33 Kwon S, Perera S, Pahor M, Katula JA, King AC, Groessl EJ, et al: What is a meaningful change in physical performance? Findings from a clinical trial in older adults (the LIFE-P study). J Nutr Health Aging 2009; 13:538-544.

34 Perera S, Studenski S, Newman A, Simonsick E, Harris T, Schwartz A, et al: Are estimates of meaningful decline in mobility performance consistent among clinically important subgroups? (Health ABC study). J Gerontol A Biol Sci Med Sci 2014;69:1260-1268.

35 Rabin R, de Charro F: EQ-5D: a measure of health status from the EuroQol Group. Ann Med 2001;33:337-343.

36 Farag I, Sherrington C, Kamper SJ, Ferreira M, Moseley AM, Lord SR, et al: Measures of physical functioning after hip fracture: construct validity and responsiveness of performance-based and self-reported measures. Age Ageing 2012;41:659-664.

37 Janssen B, Szenda A: Population norms for the EQ-5D; in Szenda A, Janssen B, Cabases J (eds): Self-Reported Population Health: An International Perspective Based on EQ-5D. Netherlands, Springer, 2014, pp 19-30. 